\title{
LE LAVARET DU LAC DU BOURGET. PRODUCTIVITÉ ET ALEVINAGE
}

\author{
par A. BATIAS \\ Ingénieur des Eaux et Forêts
}

Le lac du Bourget est actuellement le lac français qui présente la plus grande productivité. Situé à 231 mètres d'altitude, entre les derniers plis jurassiens de la montagne du Chat, de la Chambotte et le massif préalpin des Bauges (Mont-Revard), il ne couvre qu'une superficie totale de 4.432 hectares. Sa profondeur maximum est de 145 mètres et le volume de ses eaux atteint 3.620 millions de mètres cubes.

C'est un lac oligotrophe qui, tant par sa situation que par la richesse de ses eaux, permet à vingt six espèces différentes de poissons de prospérer.

Au point de vue économique, le Lavaret, Coregonus lavaretus L. est l'espèce la plus intéressante puisque ce poisson constitue plus de $60 \%$ de la pêche commerciale.

Le Lavaret est un poisson pélagique qui est capturé à une taille de 25 à 45 centimètres et à un poids de 250 à 500 grammes. Il peut exceptionnellement atteindre 50 centimètres et $1 \mathrm{~kg}$. Sa période de reproduction s'étend assez largement entre le 15 Novembre et le 31 Janvier avec un maximum entre le 10 Décembre et le $1^{\text {er }}$ Janvier.

La pêche au Lavaret est pratiquée uniquement par les pêcheurs professionnels dans quatre lots loués à la Chambre syndicale des Pêcheurs professionnels, représentant 3.513 hectares, soit $80 \%$ de l'ensemble du lac. Le cinquième restant constitue deux lots amodiés aux amateurs de Chambéry et d'Aix-les-Bains. La pêche aux engins y est interdite et le Lavaret peut s'y reproduire et s'y réfugier en toute tranquillité.

Deux sortes de filets sont utilisés pour la pêche au Lavaret :

La senne est un filet traînant formant nappe de 150 mètres de longueur, 15 à 20 mètres de hauteur à mailles de 45 millimètres, muni d'une poche à mailles de 30 millimètres. Le filet tendu entre deux bateaux est ramené à l'aide d'un treuil. La pêche à la senne est pratiquée dans le lac depuis des temps immémoriaux. Mais si, avant la guerre de 1914, cet engin pouvait être utilisé pendant toutes les périodes d'ouverture, son emploi a été limité aux mois de Juillet, Août et Septembre. Le nombre des sennes a été, en outre, ramené de six à cinq.

Le pic est un filet dormant à simple nappe, de forme rectangulaire de 120 mètres de longueur, 15 mètres de haut, à mailles de 45 millimètres. 
Il est immergé le soir et relevé le lendemain. Les Lavarets se prennent par les ouies au cours de leurs déplacements nocturnes. Le nombre des pics est actuellement limité à 72 .

Le service de la Pêche de l'Inspection de Chambéry tient depuis 1900 une statistique annuelle des captures effectuées dans le lac par les professionnels et par les amateurs. Malheureusement, les pêcheurs ne sont pas tenus, comme dans d'autres pays (Suisse), de fournir mensuellement à l'Administration l'état de leurs captures.

Les agents des Eaux et Forêts doivent établir eux-mêmes ces statistiques par trimestre, par lot et catégorie de permis, grâce aux renseignements bénévoles qu'ils peuvent recueillir auprès des pêcheurs et à des sondages effectués au moment de la lève des filets.

S'il est difficile d'apprécier l'importance des prises des quelque 4.000 amateurs qui pêchent aux lignes sur l'ensemble du lac, il est beaucoup plus aisé de suivre la pêche professionnelle qui ne groupe guère qu'une centaine d'adhérents.

Le Lavaret n'étant justiciable que du filet, nous pouvons faire confiance, tout au moins pour cette espèce, à nos statistiques.

D'autant que l'Administration possède depuis quelques années un recoupement assez précis : le relevé annuel de captures pendant les pèches exceptionnelles de fraye.

En effet, pour obtenir les œufs qui seront incubés dans les établissements de pisciculture, des géniteurs sont capturés chaque année en Décembre et Janvier. Les pécheurs sont autorisés à les vendre après prélèvement des œufs et de la laitance, mais ces poissons doivent être marqués à l'opercule d'un poinçon spécial dont l'empreinte est déposée au greffe du Tribunal. Le service de la pêche possède donc une statistique annuelle des géniteurs capturés qui confirme, pour le Lavaret, la statistique annuelle des poissons capturés.

Ces statistiques donnent-elles un reflet exact de la richesse piscicole du lac et, partant, de sa productivité ? Pas nécessairement.

En effet, l'utilisation d'engins trop nombreux à capacité pêchante trop élevée provoquera des pẻches supérieures à la production.

Par contre, une pêche extensive inférieure à la production donnera une idée inexacte de la richesse du lac.

Mais l'étude comparative des statistiques sur de longues périodes permet de se rendre compte de la richesse du peuplement piscicole et de son évolution dans le temps. Lorsque la pêche est supérieure à la production, les tonnages ne se maintiennent pas. Tôt ou tard, ainsi que nous le verrons, le rendement baisse et les statistiques reflètent cet appauvrissement.

Plus que les valeurs absolues des tonnages, l'intérêt des statistiques est de conserver approximativement les proportions entre les différentes espèces. Les pourcentages de capture fournissent ainsi un reflet fidèle de la répartition de chaque espèce dans les eaux du lac. 
ETAT DES POISSONS CAPTURÉS DE 1900 A 1953

\begin{tabular}{|c|c|c|c|c|c|c|c|}
\hline Année & $\begin{array}{c}\text { Poids } \\
\text { total } \\
\text { de } \\
\text { poissons } \\
\text { capturés }\end{array}$ & $\begin{array}{c}\text { Poids } \\
\text { des } \\
\text { Lavarets }\end{array}$ & $\begin{array}{c}\text { Lavaret } \\
\%\end{array}$ & Année & $\begin{array}{c}\text { Poids } \\
\text { total } \\
\text { de } \\
\text { poissons } \\
\text { capturés }\end{array}$ & $\begin{array}{c}\text { Poids } \\
\text { des } \\
\text { Lavarets }\end{array}$ & $\begin{array}{c}\text { Lavaret } \\
\%\end{array}$ \\
\hline $1900 \ldots$ & 26.734 & 13.523 & $51 \%$ & 1927. & 47.747 & 3.820 & $8 \%$ \\
\hline $1901 \ldots$ & 24.083 & 19.260 & $80 \%$ & 1928. & 52.353 & 2.420 & $5 \%$ \\
\hline $1902 \ldots$ & 24.209 & 18.020 & $74 \%$ & 1929. & 72.508 & 12.275 & $17 \%$ \\
\hline 1903. & 21.012 & 11.957 & $57 \%$ & 1930. & 68.159 & 5.646 & $8 \%$ \\
\hline $1904 \ldots$ & 23.722 & 9.342 & $39 \%$ & 1931. & 68.451 & 4.795 & $7 \%$ \\
\hline $1905 \ldots$ & 28.516 & 10.810 & $38 \%$ & 1932. & 74.249 & 2.365 & $3 \%$ \\
\hline $1906 \ldots$ & 28.633 & 9.077 & $32 \%$ & 1933. & 62.533 & 1.513 & $2 \%$ \\
\hline 1907. & 23.946 & 7.234 & $30 \%$ & 1934. & 48.790 & 1.910 & $4 \%$ \\
\hline 1908 . & 23.685 & 4.952 & $21 \%$ & $1935 \ldots$ & 56.897 & 1.917 & $3 \%$ \\
\hline 1909. & 18.557 & 3.533 & $19 \%$ & 1936. & 57.870 & 2.544 & $4 \%$ \\
\hline $1910 \ldots$ & 17.494 & 6.388 & $36 \%$ & 1937. & 76.224 & 3.600 & $5 \%$ \\
\hline $1911 \ldots$ & 17.925 & 4.282 & $24 \%$ & 1938. & 68.855 & 3.671 & $5 \%$ \\
\hline $1912 \ldots$ & 17.413 & 3.805 & $22 \%$ & $1939 \ldots$ & 66.819 & 3.623 & $3 \%$ \\
\hline 1913. & 16.384 & 2.894 & $18 \%$ & $1940 \ldots$ & 69.421 & 2.775 & $4 \%$ \\
\hline 1914. & 17.383 & 9.401 & $54 \%$ & $1941 \ldots$ & 64.115 & 3.087 & $5 \%$ \\
\hline 1915. & & & & 1942. & 48.000 & 1.353 & $3 \%$ \\
\hline 1916. & & & & 1943. & 49.721 & 2.980 & $6 \%$ \\
\hline 1917. & 30.065 & 12.560 & $42 \%$ & 1944. & 46.795 & 3.620 & $8 \%$ \\
\hline 1918. & 26.014 & 7.300 & $28 \%$ & 1945. & 55.570 & 7.540 & $13 \%$ \\
\hline 1919. & 31.919 & 17.650 & $55 \%$ & 1946. & 88.660 & 35.450 & $41 \%$ \\
\hline $1920 \ldots$ & 31.906 & 13.500 & $42 \%$ & 1947. & 131.097 & 87.219 & $66 \%$ \\
\hline $1921 \ldots$ & 17.573 & 7.248 & $41 \%$ & $1948 \ldots$ & 121.225 & 52.600 & $43 \%$ \\
\hline $1922 \ldots$ & 19.637 & 10.578 & $54 \%$ & $1949 \ldots$ & 150.390 & 92.150 & $61 \%$ \\
\hline 1923. & 21.296 & 10.171 & $48 \%$ & 1950. & 137.370 & 76.980 & $56 \%$ \\
\hline 1924. & 23.078 & 7.872 & $34 \%$ & $1951 \ldots$ & 139.450 & 93.500 & $67 \%$ \\
\hline 1925. & 52.356 & 18.145 & $35 \%$ & $1952 \ldots$ & 136.925 & 92.800 & $67 \%$ \\
\hline $1926 \ldots$ & 57.406 & 11.225 & $19 \%$ & $1953 \ldots$ & 161.623 & 97.708 & $60 \%$ \\
\hline
\end{tabular}

Pour étudier l'évolution de la pêche et de la productivité piscicole du lac du Bourget, nous avons divisé en cinq périodes la première moitié de ce siècle.

\section{1 re PÉRIODE 1900-1914}

La pèche est pratiquée d'une manière extensive.

Si les professionnels disposent de six sennes qu'ils utilisent toute l'année sauf en dehors des périodes d'interdiction, le pic est incomnu, la pêche au Lavaret se pratique avec des araignées à mailles de 40 millimètres, de petites dimensions puisqu'elles ne dépassent pas $1 \mathrm{~m} .20$ de hauteur et $\mathbf{4 0}$ mètres de longueur.

La pêche est, à cette époque, un métier cxlénnant. Les moteurs sont inconnus. Les pècheurs de Bourdeau et de Conjux doivent effectuer soir et matin plus de 15 kilometres en ramant pour la pose et la releve 
des filets au large de l'Abbaye d'Hautecombe. Ces longs trajets à la rame sont d'autant plus pénibles que le lac est soumis à l'action de vents violents du nord (bise) ou de l'ouest (traverse).

Une autre difficulté est l'écoulement du Lavaret. Ce poisson, à chair délicate, doit ètre consommé aussitôt que pêché car il ne survit pas à sa capture.

Pourtant les frigorifiques sont inconnus; les moyens de locomotion restent précaires et la Savoie n'a pas encore l'essor touristique qu'elle a connu depuis. Le poisson n'a donc que des débouchés locaux et restreints. Chaque jour, on voit les femmes de pêcheur apporter à Chambéry et à Aix-les-Bains, à 10 ou 15 kilomètres, les poissons que leurs maris ont eu tant de mal à capturer.

La pêche doit être largement inférieure à la production. Pourtant, les proportions entre les diverses espèces capturées reflètent assez bien la population piscicole du lac. Les amateurs sont encore peu nombreux, ils utilisent également des bateaux à rames et les Chambériens n'ont pas les moyens de locomotion nécessaires pour leur permettre de profiter de leurs moindres loisirs.

Jusqu'en 1909 , le poids total des poissons capturés ne dépasse guère 20 tonnes, dont 30 à $50 \%$ sont des Lavarets.

Il semble, cependant, qu'entre 1910 et 1914 nous assistions déjà à une régression des Corégones qui tombent à $24 \%$ puis à $18 \%$.

Peut-être faut-il l'attribuer au développement des grands carnassiers (Perches, Brochets) qui devaient, à l'époque, inquiéter le service forestier, puisqu'il organisait des pêches exceptionnelles pour détruire les Perches.

Au cours de cette période, le poids total annuel des poissons capturés a été de : $21.980 \mathrm{kgs}$, soit $5 \mathrm{kgs}$ par hectare dont $8.941 \mathrm{kgs}$ en Lavaret, soit 2 kgs par hectare.

2e PérIOde 1919-1925.

Pendant la première guerre mondiale, il n'a pas été tenu de statistique. Les hommes sont mobilisés et la pèche est, à cette époque, pratiquement nulle. Seuls, les vieillards et quelques femmes posent encore des filets pour assurer leur subsistance.

Pourtant, l'année 1917 est restée célèbre dans la mémoire des vieux pêcheurs, particulièrement pour l'abondance des prises de Lavaret.

Cet arrèt de la pêche ne manque pas de se faire sentir dès la démobilisation générale. Les Corégones ont pu se reproduire facilement au cours de ces cinq années et, dès 1919 , la pêche se révèle abondante puisque le tonnage atteint 31 tonnes dont $55 \%$ de Lavaret.

La pêche va s'intensifier, car son exercice devient de plus en plus rémunérateur. La hausse des prix, le développement du tourisme surtout en été et l'accroissement des moyens de locomotion permettent un écoulement plus aisé du poisson. 
C'est à cette époque que se vulgarise l'emploi du pic. Ce filet a été utilisé pour la première fois en 1880 par un pêcheur du lac Léman, Marie Lugrin de Meillerie. Il eut, en effet, l'idée de juxtaposer les araignées ayant 80 mètres de longueur et $1 \mathrm{~m}$. 20 de hauteur pour fabriquer des filets de 160 mètres de longueur et de 15 mètres de hauteur. Ces pics sont accouplés en longueur et en hauteur pour former de grands barrages qui permettent la capture de grandes quantités de Lavarets.

Connus avant 1914 dans le lac du Bourget, les pics ne se vulgarisèrent vraiment qu'à partir de 1919 .

L'utilisation des pics permit des captures importantes de Lavarets, et il est vraisemblable que la pêche, au cours de cette période, devait très largement dépasser la production, comme devait le prouver, par la suite, la chute brutale des rendements.

Le poids moyen annuel des captures s'éleva à $28.278 \mathrm{kgs}$, soit $6 \mathrm{kgs}$ par hectare, dont $12.166 \mathrm{kgs}$ en Lavarets et $3 \mathrm{kgs}$ par hectare.

La baisse de la pêche au Lavaret commence dès 1924. Le pourcentage des captures de cette espèce tombe à $34 \%$.

\section{3e PÉRIOde 1926-1939.}

Nous arrivons à la période la plus noire de l'histoire de la pêche dans le lac du Bourget. L'appauvrissement en Lavarets va aller en s'accentuant au fur et à mesure que les pêcheurs augmentent le nombre des pics et que la pauvreté des captures les incitent à placer leurs filets sur les frayères, aussi bien en décembre quand la pêche est interdite qu'en janvier quand la pêche est ouverte, mais que la fraye n'est pas terminée.

La vulgarisation des moteurs va permettre, en outre, d'augmenter les heures de pêche tout en diminuant la fatigue des professionnels.

Aussi, les résultats ne se font pas attendre. Le tonnage global des poissons capturés continue de s'accroître, car le nombre des pècheurs amateurs augmente d'année en année. De 57 tonnes en 1926, le rendement total du lac passe à 74 tonnes en 1932 pour se maintenir entre 65 et 75 tonnes jusqu'en 1931. La moyenne des captures s'élève à $62.776 \mathrm{kgs}$ par an, soit $14 \mathrm{kgs}$ par hectare, mais elles sont représentées surtout par des poissons blancs (Perche principalement).

Le pourcentage des Lavarets, l'année 1929 mise à part, va en continuelle décroissance : $8 \%$ en 1926, $5 \%$ en 1928, pour se stabiliser entre 3 et $5 \%$ de 1930 à 1939. En moyenne, les Lavarets ne représentent que $4.380 \mathrm{kgs}$ par an et $1 \mathrm{~kg}$ par hectare.

Émue par cette situation, l'Administration s'efforça de protéger la reproduction et le développement du Lavaret par un renforcement de la règlementation en fixant à 120 le nombre des pics, illimité jusqu'alors. 
RELEVÉ DES DEVERSEMENTS D'ALEVINS

\begin{tabular}{|c|c|c|c|}
\hline Année & $\begin{array}{c}\text { Lavaret } \\
\text { (Nombre d'alevins) }\end{array}$ & Année & $\begin{array}{c}\text { Lavaret } \\
\text { (Nombre d'alevins) }\end{array}$ \\
\hline $\begin{array}{l}1928 \ldots \ldots \ldots \\
1929 \ldots \ldots \ldots \\
1930 \ldots \ldots \ldots \\
1931 \ldots \ldots \\
1932 \ldots \ldots \\
1933 \ldots \ldots \ldots \\
1934 \ldots \ldots \\
1935 \ldots \ldots \ldots \\
1936 \ldots \ldots \\
1937 \ldots \ldots \\
1938 \ldots \ldots \\
1939 \ldots \ldots \\
1940 \ldots \ldots \\
1941 \ldots \ldots \ldots \\
\end{array}$ & $\begin{array}{r}25.000 \\
- \\
2.000 .000 \\
256.000 \\
325.000 \\
240.000 \\
375.000 \\
1.974 .000 \\
2.990 .000 \\
2.585 .000 \\
4.400 .000 \\
3.680 .000 \\
5.357 .000 \\
2.008 .000\end{array}$ & $\begin{array}{l}1942 \ldots \ldots \ldots \\
1943 \ldots \ldots \ldots \\
1944 \ldots \ldots \ldots \\
1945 \ldots \ldots \\
1946 \ldots \ldots \\
1947 \ldots \ldots \\
1948 \ldots \ldots \\
1949 \ldots \ldots \\
1950 \ldots \ldots \\
1951 \ldots \ldots \\
1952 \ldots \ldots \\
1953 \ldots \ldots \\
1954 \ldots \ldots \\
\ldots\end{array}$ & $\begin{array}{r}754.300 \\
2.151 .000 \\
980.000 \\
2.050 .000 \\
1.080 .000 \\
5.535 .000 \\
7.663 .000 \\
10.500 .000 \\
11.358 .000 \\
9.270 .000 \\
13.520 .000 \\
14.365 .000 \\
12.573 .000\end{array}$ \\
\hline
\end{tabular}

L'Administration et les pêcheurs pensèrent alors que la productivité ne pouvait être augmentée dans un lac intensément pêché que par la reproduction artificielle.

Les premiers essais datent de 1929. 600.000 œufs de Palée (Coregonus fera JuR.) furent importés du lac de Neuchatel, malheureusement par un froid très vif. L'eau des caisses d'expédition gela partiellement et il ne put être déversé que 25.000 alevins.

En 1930, le Syndicat des Pêcheurs Professionnels fit construire trois établissements pour l'incubation des œufs à Grésine, au Bourgetdu-Lac et à Conjux.

Les premiers essais furent irréguliers, car on se contentait de recueillir les œufs de géniteurs capturés en janvier après l'ouverture, en profitant de la longue période de fraye de l'espèce.

$\mathrm{Si}$, en 1930 , les déversements d'alevins s'élèvent à 2.000 .000 , soit 450 par hectare, ils tombèrent à 256.000 en $1931,325.000$ en $1932,240.000$ en 1933 et 375.000 en 1934, correspondant à une moyenne de 68 à l'hectare.

C'était insuffisant!

Aussi, après que l'École Nationale des Eaux et Forêts eut construit l'établissement d'hydrobiologie du Petit-Port à Aix-les-Bains, auquel était annexé une pisciculture permettant l'incubation des Corégones, l'Inspection de Chambéry décida d'organiser systématiquement des pêches de géniteurs avec le concours des pêcheurs professionnels.

Ceux-ci posent sur les frayères, même dans le mois de décembre, des araignées, sous la surveillance de l'Administration. Les filets sont relevés le lendemain et la fécondation est immédiatement pratiquée sur les bateaux. C'est qu'en effet, en raison de la fragilité des Corégones, 
la fécondation artificielle ne donne de bons résultats que si elle est exécutée rapidement.

On sait qu'elle est opérée dans une simple cuvette avec des œufs parfaitement murs et sans excès de laitance. Les œufs fécondés sont lavés avec soin pour éviter qu'un excès de laitance ne les agglutine en paquet, où ils seraient rapidement asphyxiés.

Les pêcheurs du lac du Bourget ayant acquis une longue pratique de la fécondation artificielle, obtiennent d'excellents rendements. La réussite à l'éclosion atteint 90 à $95 \%$.

Ces pêches exceptionnelles de géniteurs ont été pratiquées systématiquement chaque année depuis 1934. Mais les premiers résultats furent décevants. Il fallut, cette année-là, six jours de pêche sur les frayères de quatre lots du lac pour retirer $33 \mathrm{kgs}$ de géniteurs et obtenir 375.000 alevins.

Les années suivantes, la production des œufs s'améliora mais en pêchant intensément pendant toute la période de fraye.

Néanmoins, de 1935 à 1939 , une moyenne de 3.100 .000 alevins furent immergés, chaque année, représentant une densité de 700 alevins à l'hectare.

\section{4e PÉRIODE 1939-1945.}

Peut-être eût-il fallu, comme pendant la guerre de 1914-1918, négliger les cinq années de la guerre. Pourtant les pêches continuèrent à être pratiquées avec la même intensité. Pour les poissons blancs, la pêche amateur va même s'accroître puisque nombre d'entre eux vont y chercher un appoint à un ravitaillement insuffisant.

Pourtant, le tonnage global tombe de 66 tonnes à 46 tonnes en 1944 . Le service de péche a, pendant cette période, les plus grandes difficultés pour établir des statistiques. Les pêcheurs sont peu soucieux de révéler le montant de leurs captures par crainte des impositions.

Quantité de Lavarets ne figurent pas sur nos états parce qu'ils seront consommés sur place ou parce qu'ils seront nécessaires aux pêcheurs pour obtenir les filets et le chalam que mesurent chichement les services de répartition.

En 1943 et 1944, l'Administration fut obligée de faire exploiter la pêche par licences dans un village qui n'avait pas livré ses impositions. Aussi, les statistiques sous-estiment sùrement l'importance des captures.

Pourtant l'alevinage, sauf au cours de l'hiver 1939-1940, continue et ce qu'il est intéressant de noter c'est que pour la première fois depuis 1930 , le pourcentage des Lavarets capturés monte à $6 \%$ en $1943,8 \%$ en 1944 pour atteindre $13 \%$ en 1945 , dernière année d'imposition.

Un incident fortuit donne, à cette époque, aux pêcheurs un espoir en un avenir meilleur. Au cours du printemps 1944, un avion allemand désemparé lance une bombe au milieu du lac. Des milliers de Lavarets de toute taille sont tués; l'enrichissement en Corégones est commencé. 
Les pêches exceptionnelles sont encore mëdiocres. Sans doute, la plupart des alevins déversés au cours des années précédentes ont péri ou n'ont pas encore eu le temps de se reproduire.

Le Lavaret est un poisson de croissance assez lente. Un alevin fraîchement éclos met trois ans pour atteindre 20 centimètres et cinq ans pour obtenir sa taille adulte.

Les rendements des pêches exceptionnelles de Lavarets sont les suivants :

1940-1941 : 13 jours de pêche, 457 géniteurs pesant 172 kgs. Incubation de 2.382 .000 cufs.

1941-1942 : 13 jours de pêche, 651 géniteurs pesant 250 kgs. Incubation de 3.100 .000 oufs.

1942-1943 : 13 jours de pêche, 359 géniteurs pesant $150 \mathrm{kgs}$. Incubation de 2.924.000 oufs.

1943-1944: Pas de pêche.

1944-1945: 7 jours de pêche seulement en raison des inondations de décembre, 378 géniteurs pesant $150 \mathrm{kgs}$. - Incubation de 2.300 .000 œufs.

1945-1946 : 11 jours de pêche, 979 géniteurs pesant 342 kgs. Incubation de 2.300.000 œufs.

$\mathrm{Au}$ cours de cette période, la réglementation de la pêche devint plus sévère. Si le nombre des sennes resta limité à 5 , celui des pics fut ramené de 120 à 48 .

\section{5e Période 1946-1953.}

La suppression des impositions en 1945 coïncide immédiatement avec une augmentation générale des captures annuelles. Le service forestier peut, plus facilement, obtenir les renseignements dont il a besoin et les statistiques reflètent mieux la réalité. de tous.

C'est à cette époque aussi que le succès de l'alevinage éclata aux yeux

Non seulement le rendement global du lac monta en flèche pour passer de 88 tonnes en 1946 à 131 tonnes en 1947, pour se stabiliser entre 136 et 139 tonnes de 1950 à 1952, mais les pourcentages de Lavarets passèrent de 41 en 1946, à 61 en 1949, pour atteindre 67 en 1951 et 1952, soit approximativement la proportion normale donnée par KreITMaNN en 1933 pour un lac subalpin. Le lac du Bourget avait retrouvé son caractère primitif de lac à Corégones.

Les statistiques furent corroborées par les résultats des péches exceptionnelles de décembre et janvier.

1946-1947 : 16 jours de pêche, 5.172 géniteurs pesant $1.876 \mathrm{kgs}$. Incubation de 7.350 .000 œufs. 


\section{STATISTIQUE - 1900 A 1953}

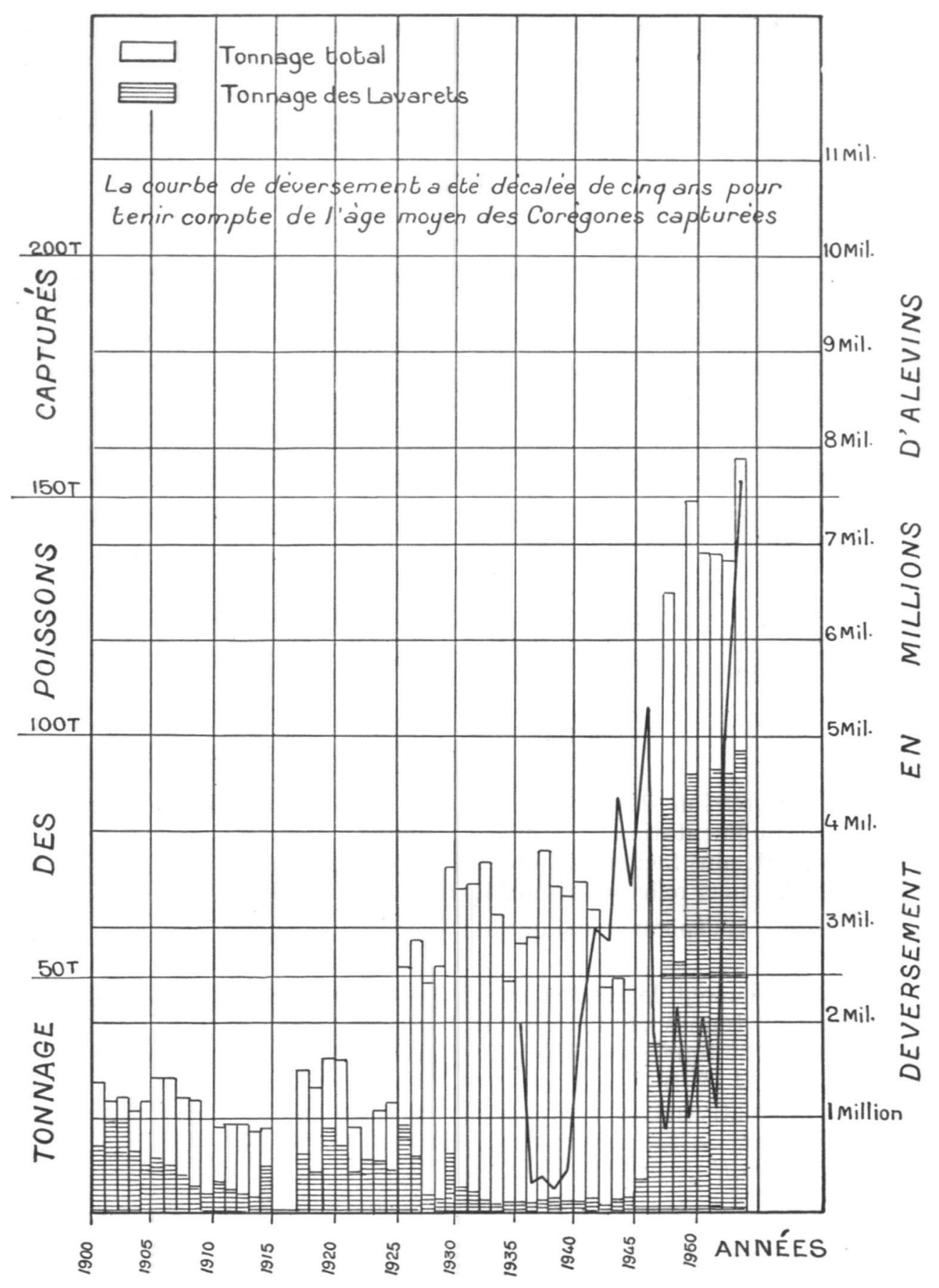


1947-1948 : 18 jours de pèche, 4.979 géniteurs pesant 2.084 kgs. Incubation de 10.315 .000 oufs.

1948-1949 : 18 jours de pêche, 9.294 géniteurs pesant 3.209 kgs. Incubation de 12.000 .000 œufs.

A cette époque, les incubations ne sont faites que dans les trois établissements du Bourget-du-Lac, de Conjux et du Petit-Port à Aixles-Bains, mais la température relativement élevée de la source alimentant les incubateurs de Conjux $\left(8\right.$ à $\left.10^{\circ}\right)$ accélère l'éclosion des œufs (30 jours) et permet de faire deux élevages de Lavaret au cours d'une même saison de pêche. La mise à l'eau du premier élevage peut être effectuée avant l'achèvement de la fraye.

En 1949, le Service forestier, devant l'abondance des pèches, n'est plus maître du contrôle de la fécondation qui, faite hâtivement ou sur des femelles retirées de l'eau depuis plusieurs heures, donne des rendements plus faibles. La réussite à l'éclosion tombe de $95 \%$ à $85 \%$.

Aussi, depuis trois ans, les pêches, au lieu d'être faites simultanément sur 4 lots, ne le sont plus que sur deux lots. Il aurait donc normalement fallu deux fois plus de jours de pêche pour obtenir les mêmes quantités de géniteurs.

Or, les résultats des pêches exceptionnelles continuent d'augmenter :

1949-1950 : 6 jours de pêche sur deux lots, 17.342 géniteurs pesant 6.091 kgs. - Incubation de 11.000 .000 d'œufs.

1950-1951 : 10 jours ds pêche, 16.014 géniteurs pesant 5.027 kgs. Incubation de 9.270 .000 œufs.

1951-1952 : 10 jours de pêche, 22.016 géniteurs pesant 7.343 kgs. Incubation de 15.000.000 d'œufs.

En 1952, la Chambre Syndicale des Pêcheurs Professionnels fit reconstruire l'établissement de pisciculture de Brinon-Saint-Innocent.

Il n'était plus utilisé depuis quelques années parce qu'il était alimenté par une source à faible débit pendant les années de sécheresse et dont les eaux étaient surchargées de calcaire.

Un nouvel établissement fut construit aux bords mêmes du lac, à Grésine, permettant l'installation de 28 carafes Zug.

Actuellement, chaque année, l'incubation des œufs de Lavaret pourra être faite dans 93 carafes, compte tenu de la possibilité d'effectuer deux élevages par saison à l'établissement de Conjux. La possibilité annuelle d'élevage est portée à 17 millions d'œufs. Ce chiffre fut d'ailleurs atteint au cours de la saison 1952-1953.

Les pêches exceptionnelles de Lavaret furent organisées comme à l'accoutumée tantôt sur les lots 1 et 4 , tantôt sur les lots 2 et 3 .

Au cours de 9 pèches entre le 9 décembre et le 21 janvier, 17.945 géniteurs ont été capturés pesant 6.447 kgs dont 13.610 mâles, 3.736 femelles mûres et 479 femelles non mûres. Le nombre d'œufs fécondés a dépassé 21 millions, dont 4 millions ont été livrés aux lacs Léman, d'Annecy et de Paladru et 17 millions ont été traités pour le lac du Bourget. 
Signalons particulièrement les pêches des 9 et 10 décembre en pleine période de frai, qui s'élevèrent à 12.564 géniteurs, soit plus de $70 \%$ des captures.

Le tonnage des géniteurs capturés souligne, peut-être encore plus éloquemment que les statistiques de pêche, la richesse actuelle du lac du Bourget en Lavarets.

Il prouve que la production globale de 150 tonnes, soit $30 \mathrm{kgs}$ par hectare dont $21 \mathrm{kgs}$ de Lavaret, n'est pas surestimée. Elle est largement supérieure au chiffre de $15 \mathrm{kgs}$ par hectare indiqué par KreItMaxi dans les lacs subalpins.

Cet éminent technicien signalait, en effet, que la production avait atteint en 1920-1922, dans le Léman; 26 kgs à l'hectare, mais il considérait cette production comme anormale, explicable seulement par un prélèvement piscicole supérieur à son accroissement.

Le Professeur LÉGER admettait que la production maximum d'un lac subalpin n'était que de $25 \mathrm{kgs}$ à l'hectare.

Il semble que dans un lac oligotrophe de dimension moyenne, comme le Bourget, un rendement supérieur puisse être obtenu sans que ce soit aux dépens du capital.

L'étude des statistiques de pêche semble indiquer que l'équilibre biologique du lac du Bourget, après avoir été rompu au cours des années 1926-1939 au détriment des Corégones, semble avoir retrouvé son caractère primitif de lac à Corégones à la suite d'alevinages massifs et continus.

La courbe de production de ce poisson prend une allure caractéristique d'S avec un palier inférieur pour les années antérieures à 1945 et un palier supérieur pour les années postérieures à 1946 .

Doit-on en conclure que la reproduction artificielle est la cause unique et absolue de cet enrichissement?

Il convient tout d'abord d'examiner de plus près les conditions de la reproduction naturelle dans le lac. Bien que le Bourget ne serve pas de bassin de compensation pour usine hydroélectrique, comme son voisin le lac d'Aiguebelette, le simple jeu des phénomènes naturels peut y provoquer des abaissements brusques de niveau.

On sait que le Lavaret se reproduit sur des frayères caillouteuses en bordure des rives, là où le ressac oxygène vigoureusement les eaux, sous quelques décimètres de fond, principalement entre le 10 décembre et le 1 er janvier.

Les géniteurs profitent des crues fréquentes provoquées à cette époque de l'année par les pluies d'automne ou les fontes de neige précoces. La température de l'eau sur les frayères est voisine de 5 a $6^{\circ}$ et la durée d'incubation des œufs de Corégones est de 45 à 50 jours.

Entre le 15 décembre et le 15 février, les pontes d'œufs fécondés sont particulièrement sensibles aux oscillations du lac. Or, celles-ci sont 
souvent fort grandes car aux pluies d'automne succède généralement une période de grands froids, provoquant des chutes de neige en montagne, la décrue des affluents du lac et une baisse des eaux. Les plages sur lesquelles reposent les œufs sont mises à sec et les œufs fécondés déposés sur les graviers périssent.

Cette action néfaste des changements de niveau pendant la période d'incubation est encore accentuée par les tempêtes fréquentes qui sévissent sur le lac dans les derniers jours de décembre (1952-1953).

Le tableau ci-après indique que la production naturelle ne réussit pleinement qu'une année sur quatre en moyenne.

RÉUSSITE DE LA REPRODUCTION NATURELLE

\begin{tabular}{|c|c|c|c|}
\hline Normale & Moyenne & Faible & Quasi nulle \\
\hline \multicolumn{4}{|c|}{$\begin{array}{c}\text { BAISSE DU NIVEAU DU LAC PENDANT LA FRAYI } \\
\text { ET L'INCUBATION DES EUFS }\end{array}$} \\
\hline $\begin{array}{c}\text { Montée du lac } \\
\text { ou baisse inférieure } \\
\text { à } 0 \mathrm{~m} .30\end{array}$ & $0 \mathrm{~m} .30$ à $0 \mathrm{~m} .50$ & $\begin{array}{l}0 \mathrm{~m} .50 \\
0 \mathrm{~m} .50 \text { à } 1 \mathrm{~m}\end{array}$ & Plus de $1 \mathrm{~m}$. \\
\hline $\begin{array}{l}1938-1939 \\
1942-1943 \\
1947-1948 \\
1948-1949\end{array}$ & $\begin{array}{l}1941-1942 \\
1943-1944 \\
1946-1947 \\
1947-1950\end{array}$ & $\begin{array}{l}1936-1937 \\
1937-1938 \\
1940-1941 \\
1951-1952\end{array}$ & $\begin{array}{l}1939-1940 \\
1944-1945 \\
1950-1951 \\
1952-1953\end{array}$ \\
\hline
\end{tabular}

La reproduction artificielle aurait l'avantage de soustraire les œufs pendant l'incubation aux dénivellations du lac, aux tempêtes et aux prédateurs (Perche, Lotte, Brochet) qui hantent les frayères.

Doit-on en conclure que l'alevinage est la cause indiscutable de l'enrichissement massif en Lavaret du peuplement piscicole du lac pendant les années 1946 et 1947 ?

L'étude comparative des courbes de capture et des déversements d'alevins ne permet pas d'établir de relations précises puisqu'aux années de forte production (1947 à 1951) correspondent des déversements plus faibles (1942-1946) qu'au cours des années antérieures. Abstraction faite des années 1952 et 1953 , il y aurait même corrélation inverse entre les pèches et l'alevinage, ce qui justifierait l'hypothèse de l'inutilité de la reproduction artificielle.

Mais alors, comment expliquer " le boom " de production de 19451947 ? Ne pourrait-on pas l'attribuer à la colonisation du lac par une espèce nouvelle introduite soit en 1929 (Palée), soit en 1934-1939 (Coré- 
gone du Léman)? Mais ces importations n'ont toujours eu qu'une importance restreinte et Dotrress a magistralement prouvé qu'aucune confusion possible ne pouvait être faite entre les diverses espèces de Corégones.

Le Lavaret du Bourget est un Corégone ayant de 31 à 44 branchiospines (moyenne 38,07) sur le premier arc branchial. Il ne peut être confondu ni avec la Palée, ni avec le Corégone du Léman dont le nombre moyen de branchiospines est voisin de 27.

Le Lavaret actuel est la même espèce que celle qui existait autrefois. Il n'y a pas eu colonisation.

D'autres hypothèses peuvent également être formulées.

L'enrichissement de 1947 correspondrait exactement à la diminution de la Perche en 1942 par suite d'une violente épidémie de "typhus " qui détruisit des quantités de perchettes. Toutefois, cette maladie est bien connue des techniciens et des pêcheurs. Elle présente une allure cyclique caractéristique. D'autres épidémies s'étaient produites auparavant, notamment en 1923 et 1938, sans qu'elles aient provoqué un enrichissement du lac en Corégones.

Enfin, il faut signaler un renforcement de la réglementation de la pêche pendant la guerre, la diminution du nombre des pics (120 jusqu'en 1941, 48 de 1942 à 1944, 60 de 1945 à 1946, 72 depuis 1947) et la limitation de la pêche à la senne à trois mois l'été.

Toutes ces hypothèses paraissent insuffisantes pour expliquer le changement brutal survenu en 1946-1947 dans la répartition des diverses espèces dans le lac du Bourget. Il semble que seul l'alevinage intensif et continu pratiqué pendant plusieurs années puisse expliquer le phénomène. Au lieu de raisonner par année, ne conviendrait-il pas de les grouper pour en tirer les conclusions suivantes?

1929-1934 : Alevinage faible et irrégulier, aucun résultat.

1935-1940 : Alevinage plus intense et continu qui ne se traduit pas dans l'immédiat par une augmentation de la production, parce qu'il est effectué dans un milieu pauvre en Lavaret et dans lequel prédominent les poissons carnassiers, la Perche au premier chef.

1941-1945 : Continuation de l'alevinage avec une moindre intensité en comparaison avec les années précédentes, mais encore suffisamment élevé puisque la moyenne annuelle des déversements atteint : 1.588 .000 , soit 360 par hectare. La production reste encore faible malgré que les données statistiques aient été faussées par la guerre. La limitation du nombre des engins entraîne également une diminution du tonnage capturé.

1946-1947 : La production augmente rapidement. Elle est représentée d'une part par la production naturelle du stock de Lavaret introduit de 1935 à 1940 et par l'alevinage de la période 1941 à 1945.

L'équilibre biologique est renversé en faveur des Corégones.

1948-1953 : Stabilisation de la production, malgré l'augmentation continuelle de l'alevinage. Il semble que la limite de productivité du lac 
ait été atteinte. On ne peut savoir si, dans ces conditions, l'alevinage est encore nécessaire. Les déversements sont actuellement de 10 à 15 millions par an, soit 2 à 3.000 alevins par hectare.

En résumé, nous dirons que l'alevinage artificiel a joué un rôle capital pour le réempoissonnement en Corégones du lac du Bourget, mais qu'il semble n'avoir aucun effet :

10 Dans une période d'extrême pauvreté, quand l'équilibre biologique est rompu en faveur des espèces carnassières.

$2^{\circ}$ Dans une période de grande richesse, lorsque les déversements sont supérieurs à la capacité de production et que la reproduction naturelle suffit à maintenir l'équilibre biologique.

Un enseignement doit être retiré : l'alevinage artificiel ne peut donner des résultats dans un milieu appauvri que s'il est MASsIF et poursuivi pendant de longues années.

\section{BIBLIOGR A PHIE}

Dottrens (E.) et Quartier (A.). - "Les Corégones du lac de Neuchâtel. Etude biométrique ". - Revue Suisse de Zoologie 56, 689, 1949.

Dottrens (E.). - "Le Corégone actuel du Léman". - Revue Suisse de Zoologie, $57,789,1950$.

Dottrens (E.). - "Sur le Lavaret du lac du Bourget ". - Archives des Sciences, 3, 189, 1950.

Kreitmann (L.). - "Aménagement piscicole des lacs". - Revue des Eaux et Forêts. - Janvier 1933.

VAN Oosten (J.). - " Relationship between the plantings of fry, and production of Whitefish in Lake ERIE ". - Transm. Am. Fish. Soc., 71, 118, 1941.

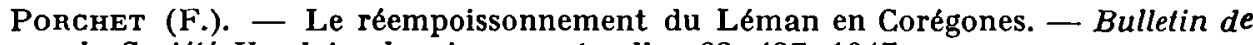
la Société Vaudoise de sciences naturelles, 63, 427, 1947.

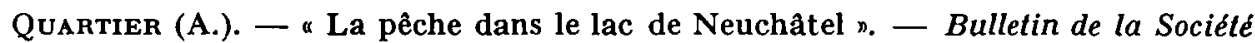
Neuchateloise de géographie.

Vibert (R.). - "Les repeuplements en Corégones ». - Extrait d'un ouvrage en préparation. 